\title{
INVESTIGACIÓN EN CUIDADOS EN EL ÁMBITO ASISTENCIAL: BINOMIO CLAVE PARA LA APLICACIÓN DE LA MEJOR EVIDENCIA
}

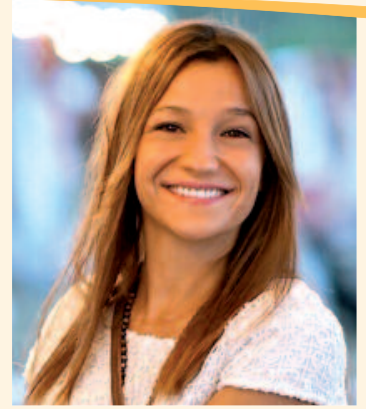

\section{MIRIAM BRONCANO BOLZONI}

Enfermera. Red de Salud Mental y Adicciones del Institut d'Assistència Sanitària. Girona.

Profesora asociada.

Departamento de Enfermería.

Universitat de Girona.

Vicepresidenta de la Associació Catalana d'Infermeria en Salut Mental (ASCISAM).
Ocho años atrás, al inicio de mi desarrollo profesional como enfermera asistencial, se gestó en mí la inquietud por la investigación. Habitualmente, se habla de «investigación», pero no siempre se entiende la globalidad del concepto y, muchas veces, se escoge como término para definir las actividades relacionadas con el mundo del laboratorio, de los ensayos clínicos, nuevos medicamentos, etc. Sin embargo, su naturaleza engloba muchos más aspectos.

El primer proyecto de investigación de la carrera me llevó a adentrarme en un pequeño estudio en el hospital. Este despertó en mí una gran inquietud por explorar la relación entre el bienestar, la recuperación y el insight de las personas con trastorno psicótico. Después de iniciar los estudios de doctorado y algún proyecto de mayor envergadura, me otorgaron una ayuda de intensificación de la investigación como enfermera: Pla Estratègic de Recerca i Innovació en Salut (PERIS) 2016-2020². Una trayectoria que inicié con el objetivo de poder transferir los resultados basados en la evidencia a mi labor asistencial.

La investigación en enfermería ha evolucionado en las últimas décadas de forma sustancial, debido, principalmente, a la evolución de los distintos planes de formación de enfermería. Por otro lado, ha habido un reconocimiento de la capacidad investigadora de las enfermeras incorporándolas en investigaciones competitivas financiadas por el Fondo de Investigación Sanitaria (FIS), también con la inclusión como investigador principal en las solicitudes de ayuda para proyectos de investigación de este colectivo².

Ha significado un importante paso adelante la realización de estudios de posgrado, máster y doctorado, así como la regulación de los títulos de especialistas en Enfermería, que actualmente dan la oportunidad de acceder al doctorado directamente (dos años de residencia). 
En todos estos procesos, ha cobrado importancia el desarrollo de los aspectos fundamentales del proceso de investigación a través de la realización de proyectos.

La enfermería es una disciplina joven; su desarrollo y afianzamiento, como en cualquier disciplina científica, están siendo impulsados por la investigación. Esta le da un carácter autónomo y un cuerpo de conocimientos propio basado en la prestación de cuidados.

La disciplina necesita fortalecerse en el ámbito de la investigación: impartir formación en investigación, incrementar la investigación a diferentes niveles y potenciar la integración de los hallazgos de la investigación en nuestra práctica clínica diaria (basados en resultados válidos y fiables siempre provenientes de investigaciones rigurosas).

La responsabilidad que tenemos desde el ámbito asistencial es garantizar que los cuidados que realizamos provienen de la mejor evidencia posible, de manera que todos formamos parte del proceso: unos profesionales producen investigación; otros resumen esta evidencia, facilitando, así, el acceso a la mejor; y otro colectivo (mucho más numeroso y experto en los cuidados en esos campos) la consume. Todo ello con la finalidad de impactar positivamente en resultados de salud en las personas que cuidamos.

Son muchos los facilitadores que a lo largo de los años han ido dirigiéndonos hacia aplicar los resultados basados en la evidencia en la práctica asistencial:

- Motivación personal por mejorar, avanzar, innovar, etc.

- Colaboración directa entre organismos financiadores e instituciones del ámbito sanitario.

- Asistencia a congresos, jornadas, etc. desde nuestros centros de trabajo en los que se comparten conocimientos.

- Fomento de la investigación de los organismos gubernamentales a través de acciones como la intensificación de la investigación en tu área de interés. Por ejemplo, las becas PERIS.

- Formación y difusión en práctica clínica basada en la evidencia.

- Difusión de las prácticas basadas en la evidencia entre los responsables, gestores, políticos, etc. La Registered Nurses' Association of Ontario (RNAO) ha lanzado una guía llamada Adopting eHealth Solutions: Implementation Strategies, que pretende aportar recomendaciones basadas en la evidencia para la puesta en marcha de proyectos relativos a la salud. Tal y como exponen en la introducción, la guía solo pretende difundir prácticas basadas en la evidencia entre los responsables, gestores, políticos, etc. que deseen poner en marcha proyectos de este tipo ${ }^{3}$.

Asistencial, docente, de gestión o de investigación. Todos y cada uno de nosotros, como profesionales de la enfermería, confeccionamos la línea de investigación que nos guía hacia un mejor cuidado de los pacientes.

\section{BIBLIOGRAFÍA}

1. Generalitat de Catalunya, Departament de Salut. Pla estratègic de recerca i innovació en salut 2016-2020. 1 . $^{\text {a }}$ ed. Barcelona: Direcció General de Recerca i Innovació en Salut; 2016. Disponible en: http://salutweb.gencat.cat/web/. content/home/ambits_tematics/linies_dactuacio/recerca/enllacos/PERIS_2016_2020.pdf

2. Abad-Corpa E, Gonzalez-Maria E, Moreno-Casbas T. Del fomento de la investigación en cuidados a la implantación de resultados en la práctica clínica. Rev Cient Soc Esp Enferm Neurol [Internet]. 2014;39(1):2-4. Disponible en: http://www.elsevier.es/es-revista-revista-cientifica-sociedad-espanola-enfermeria-319-articulo-del-fomento-investigacion -cuidados-implantacion-S2013524614000087

3. Registered Nurses' Association of Ontario (RNAO). Adopting eHealth Solutions: Implementation Strategies. [Internet]. Toronto: RNAO; 2017. Disponible en: http://rnao.ca/sites/rnao-ca/files/bpg/Adopting_eHealth_Solutions_WEB_FINAL.pdf 